\title{
The vacuolar-ATPase modulates matrix metalloproteinase isoforms in human pancreatic cancer
}

\author{
Chuhan Chung ${ }^{1,2, *}$, Christopher C Mader ${ }^{3, *}$, John C Schmitz ${ }^{4}$, Jorunn Atladottir ${ }^{2}$, Phillip Fitchev ${ }^{5}$, \\ Mona L Cornwell ${ }^{5}$, Anthony J Koleske ${ }^{6}$, Susan E Crawford ${ }^{5}$ and Fred Gorelick ${ }^{1,2}$
}

The vacuolar-ATPase (v-ATPase) is a proton transporter found on many intracellular organelles and the plasma membrane (PM). The v-ATPase on PMs of cancer cells may contribute to their invasive properties in vitro. Its relevance to human cancer tissues remains unclear. We investigated whether the expression and cellular localization of v-ATPase corresponded to the stage of human pancreatic cancer, and its effect on matrix metalloproteinase (MMP) activation in vitro. The intensity of v-ATPase staining increased significantly across the range of pancreatic histology from normal ducts to pancreatic intraepithelial neoplasms (PanIN), and finally pancreatic ductal adenocarcinoma (PDAC). Low-grade PanIN lesions displayed polarized staining confined to the basal aspect of the cell in the majority (86\%) of fields examined. High-grade PanIN lesions and PDAC showed intense and diffuse v-ATPase localization. In pancreatic cancer cells, PM-associated v-ATPase colocalized with cortactin, a component of the leading edge that helps direct MMP release. Blockade of the v-ATPase with concanamycin or short-hairpin RNA targeting the $V_{1} E$ subunit reduced MMP-9 activity; this effect was greatest in cells with prominent PM-associated v-ATPase. In cells with detectable MMP-2 activities, however, treatment with concanamycin markedly increased MMP-2's most activated forms. V-ATPase blockade inhibited functional migration and invasion in those cells with predominantly MMP-9 activity. These results indicate that human PDAC specimens show loss of v-ATPase polarity and increased expression that correlates with increasing invasive potential. Thus, v-ATPase selectively modulates specific MMPs that may be linked to an invasive cancer phenotype.

Laboratory Investigation (2011) 91, 732-743; doi:10.1038/labinvest.2011.8; published online 21 February 2011

KEYWORDS: MMP; PanIN; pancreatic cancer; v-ATPase

Cancer cells often exist in hypoxic conditions owing to unchecked growth and a deficient blood supply. ${ }^{1}$ The acidic tumor microenvironment that results from hypoxia evokes compensatory changes in the cancer cell that confer survival and growth advantages. One such mechanism is the activity of the vacuolar-ATPase (v-ATPase). ${ }^{2,3}$ This ubiquitous proton transporter mediates multiple $\mathrm{pH}$-dependent intracellular processes including vesicular trafficking, coupled ion gradient-molecular transport, and protease activation. ${ }^{4-7}$ The v-ATPase was originally characterized in association with intracellular organelles, but is now also recognized as a component of plasma membranes (PMs) that mediates proton extrusion in some normal and malignant cells. ${ }^{8-11}$
In breast cancer cells, the abundance of v-ATPases on the PM correlates with an invasive phenotype. ${ }^{11}$ Further, v-ATPase inhibitors reduce cell migration in cancer cells with high levels of PM v-ATPase. ${ }^{11}$ It has been postulated that cell surface v-ATPase activity can provide a localized proton flux creating an acidic extracellular microenvironment. ${ }^{3}$ One potential effect of this activity is to optimize $\mathrm{pH}$ for extracellular protease activation, thereby facilitating matrix degradation and cellular invasion.

The v-ATPase is comprised of two large, multi-subunit complexes organized into a soluble domain $\left(\mathrm{V}_{1}\right)$ responsible for ATP hydrolysis and a membrane-bound domain $\left(\mathrm{V}_{0}\right)$ that forms the pore allowing for proton transport across

\footnotetext{
${ }^{1}$ Department of Medicine, Section of Digestive Diseases, VA CT Research, VA CT Healthcare System, Yale University School of Medicine, West Haven, CT, USA; ${ }^{2}$ Yale University School of Medicine, New Haven, CT, USA; ${ }^{3}$ Department of Cell Biology, Yale University School of Medicine, New Haven, CT, USA; ${ }^{4}$ Section of Oncology, VA CT Healthcare System, New Haven, CT, USA; ${ }^{5}$ Department of Surgery, NorthShore Research Institute, University of Chicago Pritzker School of Medicine, Chicago, IL, USA and ${ }^{6}$ Department of Molecular Biophysics and Biochemistry, Yale University School of Medicine, New Haven, CT, USA

Correspondence: Dr C Chung, MD, Department of Medicine, Section of Digestive Diseases, VA CT Research, VA CT Healthcare System, Yale University School of Medicine, GI Research Building No. 3, West Haven, CT 06516, USA.

E-mail: chuhan.chung@yale.edu
}

*These two authors contributed equally to this work. 
membranes. ${ }^{12}$ Multiple isoforms of the 'a' subunit that comprise the $\mathrm{V}_{0}$ complex exist. Distinct $\mathrm{V}_{0}$ a isoforms are thought to be critical for targeting the $\mathrm{V}_{0}$ complex to distinct cellular membranes. For example, the $\mathrm{V}_{0} \mathrm{a} 3$ subunit on apical PMs of osteoclasts generates the acidic extracellular microenvironment required for bone resorption. ${ }^{13,14}$ Mutations in $\mathrm{V}_{0} \mathrm{a} 3$ result in the human autosomal recessive disease, osteopetrosis, which is characterized by excess bone and severely impaired osteoclast activity. ${ }^{15,16}$ A mouse model of $\mathrm{V}_{0} \mathrm{a} 3$ deletion results in severe skeletal deformities and early death, and can be averted by $\mathrm{V}_{0} \mathrm{a} 3$ gene restoration. ${ }^{17,18}$ In breast cancer cells, the $\mathrm{V}_{0} \mathrm{a} 3$ and $\mathrm{V}_{0} \mathrm{a} 4$ isoforms were also recently shown to be essential for cellular invasion. ${ }^{19}$ These findings show that specific $\mathrm{V}_{0}$ a isoforms provide a mechanism to target v-ATPase activities to the cell surface and affect cell function. These findings suggest that differences in the v-ATPase localization assessed by immunolabeling could be used to predict the behavior of cancer types, and that its use in clinical specimens could serve as a histological marker of aggressiveness.

Expression and localization of v-ATPase in human cancer specimens has not been extensively explored. To determine whether elevated v-ATPase staining corresponds to the invasive phentoype in human pancreatic cancer, we assessed the range of tissues from non-invasive pancreatic intraepithelial neoplasia (PanIN) lesions to pancreatic ductal adenocarcinoma (PDAC). Here, we report that the v-ATPase in human PDAC loses its polarity with increasing invasive potential. Furthermore, we observed that select v-ATPase isoforms are found on human pancreatic cancer cells, and that the v-ATPase localizes with known components of the cellular invasion apparatus and has functional effects on matrix metalloproteinase (MMP) activation.

\section{MATERIALS AND METHODS Human Tissue}

Archival specimens were obtained from patients who underwent surgery for a diagnosis of PDAC. The pathological diagnosis confirmed PDAC in all cases $(n=16)$. Fifty random normal ducts, PanIN lesions, and PDAC lesions were evaluated independently by two pathologists (SEC, PF). Intensity of staining was scored as $1+$ (mild), $2+$ (moderate), or $3+$ (intense). Immunolabeling was characterized as basal, mixed basal/apical, or mixed basal/diffuse. The Institutional Review Board of the VA CT Healthcare System approved the study.

\section{Antibodies and Reagents}

Antibodies to $\mathrm{V}_{1} \mathrm{E}$ (Genway), $\mathrm{V}_{0} \mathrm{a} 2$ and $\mathrm{V}_{0} \mathrm{a} 3$ (gift from $\mathrm{Dr}$ Beth S Lee, Ohio State School of Medicine) were used to assess v-ATPase isoform specificity. Antibodies to cell surface markers E-cadherin (BD Biosciences) and epidermal growth factor receptor (Cell Signaling) were used to delineate localization of v-ATPase on PMs. An anti-cortactin antibody (AbCam) was used to mark cellular invasive fronts. ${ }^{20,21}$
Secondary fluorescent antibodies were purchased from Invitrogen. Chemical reagents were purchased from Sigma.

\section{Cell Culture}

The human pancreatic cancer cell lines Panc-1, MiaPaCa, and BXPC3 were maintained according to ATCC guidelines. As $\mathrm{v}$-ATPase assembly is glucose dependent, ${ }^{22,23}$ DMEM with low $(1 \mathrm{~g} / \mathrm{l})$ and high $(4.5 \mathrm{~g} / \mathrm{l})$ glucose were used to assess the role of v-ATPase on protease activation. To obtain conditioned medium (CM), cells were grown to $80 \%$ confluence, washed twice with serum-free media, and then incubated with serum-free media overnight. CM was obtained after 18-20 $\mathrm{h}$ and concentrated approximately 40 -fold using Amicon Ultra centrifugal filters (Millipore) with a $10 \mathrm{kDa}$ cutoff.

\section{Short-Hairpin RNA Knockdown of V-ATPase Subunit, V $\mathbf{1}$ E} Oligonucleotide targeting sequences corresponding to the coding regions of human $\mathrm{V}_{1} \mathrm{E}$ were annealed and ligated into pSuper.retro.puro (Oligoengine) (Supplementary Table 1). Panc-1 cells were transfected with adeno-associated viral vector and transfected clones selected with puromycin $(1-2.5 \mu \mathrm{g} / \mathrm{ml})$. Surviving clones were maintained in puromycin $2.0 \mu \mathrm{g} / \mathrm{ml}$. After immunoblotting $\mathrm{V}_{1} \mathrm{E}$, percent knockdown was assessed by densitometry using the NIH Image J software.

\section{Immunohistochemistry and Immunofluorescence}

Immunohistochemistry was performed as described. ${ }^{24} \mathrm{Sec}-$ tions were deparaffinized, treated to inhibit endogenous peroxidase, and subjected to antigen retrieval. Slides were washed in tris-buffered saline and incubated with primary antibodies. Sections were washed, incubated with biotinylated anti-serum, and then with streptavidin complexed with horseradish peroxidase followed by diaminobenzidine. Sections were then counter-stained with hematoxylin and eosin.

For immunofluorescence labeling, pancreatic cancer cells were grown on methanol-treated coverslips. Cells were rinsed with phosphate-buffered saline, permeabilized with $0.05 \%$ saponin for $15 \mathrm{~min}$, and blocked in 3\% BSA. Coverslips were incubated with primary antibody, and then corresponding secondary antibodies. Slides were mounted with Prolong Gold with DAPI (Invitrogen). Control slides were incubated in secondary antibody only. Slides were examined with a Zeiss Axiophot immunofluorescence microscope. Images were obtained with SPOT software and overlay images obtained using Adobe Photoshop, version 9.0.

\section{Zymography and Immunoblotting}

MMPs were first cloned as cancer-specific genes and play a critical role in tumor invasion and metastases. ${ }^{25}$ To detect MMP-2/9 activities in pancreatic cancer secretions, zymography was performed using commercial (Invitrogen) 10\% gelatin-containing gels. Briefly, 10-20 $\mu \mathrm{g}$ of cellular proteins were subject to non-denaturing electrophoresis as described. ${ }^{26}$ Gels were washed in $2.5 \%$ Triton X-100 and then 
incubated in developing buffer. After Coomassie blue staining, gels were destained and the amount of MMP activity detected as clear bands was analyzed by densitometry. The purified intermediately active form $(66 \mathrm{kDa})$ of MMP-2 (5-10 ng; Calbiochem) was used as a positive control. Optimal incubation times were as follows: Panc-1 (12-16h); MiaPaCa (18-24h); and BXPC3 (6-8h), $n=4$ experiments per group. The protease activity was estimated using the $\mathrm{NIH}$ Image J software as follows: a measurement box was placed over cleared areas of degradation and the immediate nondegraded background. Values were normalized to the highest density and comparisons were made within low and high glucose conditions, and then across the different glucose concentrations.

Immunoblotting was performed as described. ${ }^{24}$ Protein content was determined by Bradford assay. After blocking in a $5 \%$ milk solution, membranes were incubated overnight with antibodies to PEDF. After washing in TBS and $0.05 \%$ Tween, the primary antibody was labeled using a peroxidaseconjugated goat anti-rabbit IgG. Peroxidase was detected by a chemiluminescence assay (Pierce). Equivalence of loading was confirmed using $\beta$-actin (Sigma) or Coomassie stain of CM.

\section{Invasion and Migration Assays}

An agarose-spot assay assessing cellular invasion number and depth was performed as described. ${ }^{27}$ Agarose spots were plated on recessed collagen-coated cell culture plates in the presence of epidermal growth factor (R\&D Systems) at a final concentration of $30 \mathrm{ng} / \mu \mathrm{l}$. Cells were plated for $4-6 \mathrm{~h}$ and allowed to adhere in the presence of normal growth medium. The medium was then changed to serum-free media for 12 $24 \mathrm{~h}$ in the absence and presence of concanamycin $50 \mathrm{nM}$. Cells that were able to invade across the outer rim of the agarose spot were counted as positive. Ten randomly selected fields under $10 \times$ for each condition were visualized.

Wound migration assays were performed as described. ${ }^{11}$ In brief, a $2 \mathrm{~mm}$ horizontal wound was created after cells were grown to confluence. The cells were washed and placed under serum-free conditions in the absence and presence of concanamycin $(50 \mathrm{nM})$. Cells were evaluated at $24-48 \mathrm{~h}$ for migration across the wound. Cells were fixed and 10 random images were captured. In each image, three random measurements of the diameter were carried out using the NIH Image J software, averaged, and values normalized based on the largest diameter. Assays were carried out in triplicate.

\section{Statistical Analysis}

$P$-values were calculated using two-tailed Student's $t$-tests on Prism software version 4.0, with $P<0.05$ deemed statistically significant. For differences in immunohistochemistry density, one-way analysis of variance was used. Values were stated as mean \pm s.e.m. or s.d.

\section{RESULTS}

Human PDAC Tissues Show Increased V-ATPase Levels and Loss of Polarity with Advancing Neoplastic Features Histological analysis from normal ducts through PanIN lesions and finally to PDAC showed distinct patterns of $\mathrm{V}_{1} \mathrm{E}$ subunit staining that differed in intensity and distribution. In the absence of a primary antibody, there was little labeling (Figure 1a). Using an antibody targeting the $\mathrm{V}_{1} \mathrm{E}$ subunit, normal ducts (Figure 1b) displayed weak staining with a mixed basal/apical (estimated average ratio intensity to negative control: $1.24 \pm 0.43$ ) pattern. Low-grade PanIN (Figure 1c) lesions showed a typical columnar appearance with basally oriented nuclei, an increased N/C ratio, and the presence of mild nuclear atypia. These lesions showed more intense v-ATPase immunoreactivity than normal ducts (intensity: $1.72 \pm 0.45$ ). Further, low-grade PanIN lesions revealed striking differences in the distribution of v-ATPase E subunit compared with apical and basal staining in normal ducts. Thus, in $86 \%$ of the fields, labeling in low-grade PanIN lesions was basally oriented with the remaining 14\% showing mixed basal/apical distribution. High-grade PanIN (Figure 1d) lesions displayed even more intense cellular labeling than the lower grade lesions (intensity: $2.56 \pm 0.50$ ), but its distribution was more diffuse than observed with lower grade lesions. (Additional images contrasting low-grade from highgrade PanIN are provided in Supplementary Figure 1.) PDAC (Figures 1e-g), whether from the primary tumor (Figure 1e), or metastatic to lymph nodes (Figure 1g) showed uniformly intense staining and diffuse labeling. Finally, as endothelial and stromal cells release proteases and contribute to tumor spread, areas of stroma were also examined for v-ATPase labeling. Positive $\mathrm{V}_{1} \mathrm{E}$ labeling that corresponded to vascular structures (Figure 1h, circular areas) or spindle-shaped cells that likely reflect fibroblasts (Figure 1h, arrows) was present in tumor-associated stroma, but not in stroma adjacent to normal ducts (Figure 1b). Findings from our histological analysis are summarized in Table 1 . These results indicate a significantly increased intensity $(P<0.001)$ and a loss of v-ATPase polarity that occurs from low-grade PanIN to invasive PDAC. The findings are consistent with in vitro studies that illustrate $\mathrm{v}$-ATPase function is necessary for cancer cell homeostatic and invasive properties. ${ }^{11,19}$

\section{Pancreatic Cancer Cells Display V-ATPase Localization and Colocalize with Components of Cellular Invasion}

Analogous to studies in other cancer cells, ${ }^{11,19} \mathrm{v}$-ATPase isoforms were evident on the PMs of some pancreatic cancer cells, whereas others displayed minimal localization on or near the PMs. Well-differentiated BXPC3 cells, which have been described as the least invasive of the three cell lines used, ${ }^{28}$ displayed far less prominent PM staining of the v-ATPase a3 isoform (Figure 2a) than that found on Panc-1 cells (Figure $2 \mathrm{~b}$ ). Similar to the $\mathrm{a} 3$ isoform, the soluble $\mathrm{V}_{1} \mathrm{E}$ isoform showed little PM localization (Figure 2c) in BXPC3 cells. To further characterize these differences, colocalization 

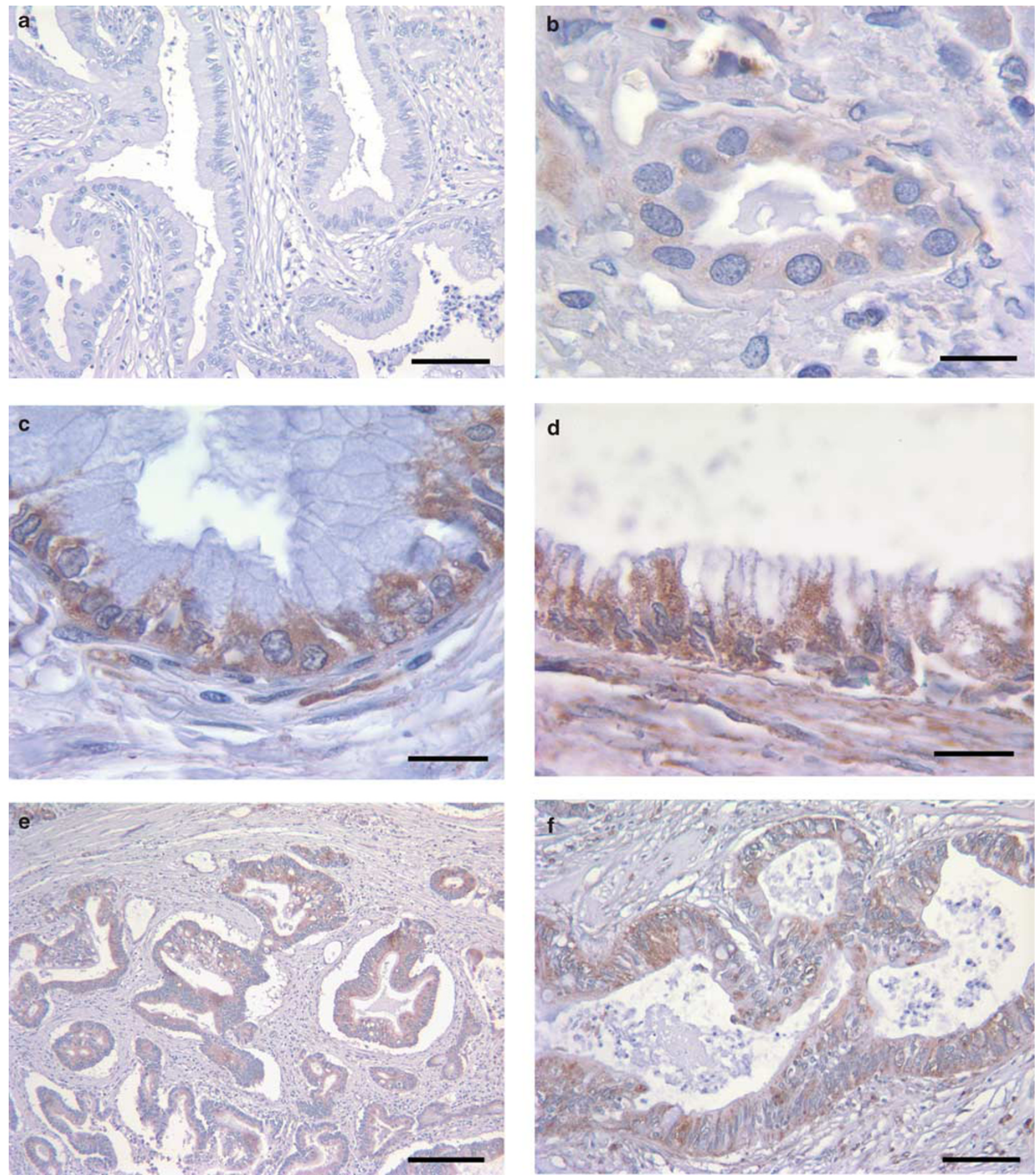

Figure 1 Immunolabeling of vacuolar-ATPase (v-ATPase) shows increased intensity and loss of polarity that corresponds to the grade of human pancreatic cancer. Labeling of the $V_{1} E$ subunit in: (a) Negative control. (b) A normal pancreatic duct shows faint staining of the v-ATPase with a mixed basal/apical distribution. (c) Low-grade pancreatic intraepithelial neoplasms (PanINs) display a more intense and polarized (basal) distribution of v-ATPase than normal cells. (d) High-grade PanIN lesions show increased irregularity in the distribution of v-ATPase within cells compared with low-grade PanIN lesions. (e) Pancreatic ductal adenocarcinoma (PDAC) (low power) and (f) PDAC (high power) show loss of polarization, diffuse but heterogeneous distribution of v-ATPase reactivity, and more intense staining than normal cells or PanIN lesions. (g) PDAC within a lymph node shows diffuse and robust staining within the cancer cells. (h) Tumor-associated stroma reveals v-ATPase labeling within endothelial cells (circular structures) and spindle-shaped cells (arrows) that likely represent tumor-associated fibroblasts. Scale bar: a, e, g, $200 \mu \mathrm{m} ; \mathbf{b}, \mathbf{C}, \mathbf{d}, \mathbf{f}, \mathbf{h}, 80 \mu \mathrm{m}$. 



Figure 1 Continued.

Table 1 v-ATPase immunolabeling shows a progressive increase in intensity and loss of polarity from early PanIN lesions to PDAC

\begin{tabular}{lcccc}
\hline Histology & $1+$ & $2+$ & $3+$ & Intensity score (mean \pm s.d.) \\
\hline Normal ducts & $38 / 50(76)$ & $12 / 50(24)$ & $0 / 50$ & $1.24 \pm 0.43$ \\
Low-grade PanIN & $14 / 50(28)$ & $36 / 50(72)$ & $0 / 50$ & $1.72 \pm 0.45$ \\
High-grade PanIN & $0 / 50$ & $22 / 50(44)$ & $28 / 50(56)$ & $2.56 \pm 0.50$ \\
PDAC & $0 / 50$ & $0 / 50$ & $50 / 50(100)$ & $3.00 \pm 0.00$ \\
\hline
\end{tabular}

\begin{tabular}{lccr} 
Histology & Basal & Mixed basal/apical & Diffuse \\
\hline Normal ducts & $0 / 50$ & $50 / 50(100)$ & $0 / 50$ \\
Low-grade PanIN & $43 / 50(86)$ & $5 / 50(10)$ & $2 / 50(4)$ \\
High-grade PanIN & $3 / 50(6)$ & $0 / 50$ & $47 / 50(94)$ \\
PDAC & $0 / 50$ & $0 / 50$ & $50 / 50(100)$
\end{tabular}

Abbreviations: PDAC, pancreatic ductal adenocarcinoma.

Immunohistochemical staining of v-ATPase was graded by two independent pathologists based on intensity (upper panel) and distribution within the cells (lower panel) in randomly selected fields. The intensity score for normal ducts (1.24 \pm 0.43 ), low-grade PanINs (1.72 \pm 0.45$)$, high-grade PanINs (2.56 \pm 0.50$)$ and PDAC (all $3+)$ were significantly different among the groups, $P<0.001$. Although the majority of normal pancreatic ducts showed a faint mixed basal/apical v-ATPase pattern, low-grade PanINs mostly (86\%) had a basal v-ATPase distribution within cells. The majority (94\%) of high-grade PanINs and all the PDAC had a diffuse distribution of v-ATPase compared with low-grade PanIN lesions, $P<0.001$.

with E-cadherin was performed. BXPC3 cells, as described previously, showed faint E-cadherin expression when plated at low density (data not shown). In culture conditions approaching confluence, E-cadherin was discernible at sites of cell-to-cell contact (Figure 2d, arrows), and showed no discernible overlap with $\mathrm{V}_{1} \mathrm{E}$. In contrast, the leading edge of Panc-1 cells exhibited $\mathrm{PM} \mathrm{V}_{1} \mathrm{E}$ staining (Figure 2e) that overlapped with E-cadherin labeling on PMs (Figure 2f, arrows), but not at cellular junctions (Figure 2f, arrowheads). (Additional high magnification images of $\mathrm{V}_{1} \mathrm{E}$ and $\mathrm{E}$-cadherin labeling in Panc-1 cells are included in Supplementary Figure 2.) Staining for $\mathrm{V}_{1} \mathrm{E}$ and epidermal growth factor receptor in Panc-1 cells (Figure $2 \mathrm{~g}$ and $\mathrm{h}$ ) further showed that the v-ATPase is present on PMs (arrows). The hypoxia mimetic cobalt chloride $200 \mu \mathrm{M}$ did not appear to increase the degree of v-ATPase labeling on PMs in Panc-1 cells (data not shown). Thus, the localization of the v-ATPase on PMs may reflect the functional ability to acidify local peri-cellular domains and contribute to the invasive phenotype of pancreatic cancer cells.

Specific regions of cancer cells reflecting the leading edge or invasive front are responsible for focal protease release required for matrix degradation and cell invasion. ${ }^{29}$ We investigated whether the v-ATPase colocalizes with other proteins that are know to reside in the leading edge. Cortactin is a prominent tyrosine kinase substrate that produces an actin assembly complex required for actin-based protrusions at the leading edge and directs MMP release at invasion sites. ${ }^{20,21,30}$ In Panc-1 cells, $\mathrm{V}_{1}$ E labeling (Figure 3a) occurred on PMs (arrow) and intracellular organelles (arrowhead). 
Cortactin localized to well-defined patches at the PM (Figure 3b). Double-labeling studies showed that the v-ATPase $V_{1} E$ subunit was located at or adjacent to regions of cortactin immunoreactivity (Figure 3c, arrow), with no overlap with cortactin staining on intracellular organelles (Figure $3 \mathrm{c}$, inset, arrowhead). These results indicate that the v-ATPase on PMs co-distributes with known components of the cellular invasion apparatus. (Cortactin colocalization with another $\mathrm{v}$-ATPase subunit, $\mathrm{V}_{0} \mathrm{a}$ 3, is shown in Supplementary Figure 3.)


Pancreatic Cancer Cell-Derived MMP-9, but not MMP-2, Activities are Decreased with V-ATPase Blockade

To examine the functional effect of $\mathrm{v}$-ATPases on MMP activities, zymography of CM was performed with chemical inhibitors and short-hairpin (sh)RNA-mediated knockdown of the v-ATPase. MMP-9 activity was present in the three pancreatic cancer cell lines examined. Incubation with concanamycin (50 nM) led to significantly decreased MMP-9 activity in each cell line; this effect was most evident in


Figure 2 Pancreatic cancer cells display plasma membrane localization of vacuolar-ATPase (v-ATPase) isoforms and show colocalization with cell surface markers. Immunofluorescent (IF) images of $\mathrm{V}_{0}$ a3 subunit labeling in (a) BXPC3 and (b) Panc-1 cells reveals that localization to plasma membranes is greater in some pancreatic cancer cells than others. Labeling of the $V_{1} E$ subunit (c) and the cell surface marker E-cadherin (d, arrows) in BXPC3 cells shows minimal colocalization. Panc-1 cells display cell surface labeling for $\mathrm{V}_{1} \mathrm{E}(\mathbf{e})$ and $\mathrm{E}$-cadherin with areas of colocalization (f, arrows) on the leading edge of cells, whereas cell-to-cell adhesions stained only for $\mathrm{E}$-cadherin ( $\mathbf{f}$, arrowheads). Colocalization of $\mathrm{V}_{1} \mathrm{E}$ (g, arrows) in Panc- 1 cells was also seen with other cell surface markers such as epidermal growth factor receptor (EGFR)-1 (h). Scale bar: $10 \mu \mathrm{m}$. 



Figure 2 Continued.

Panc-1 and MiaPaCa cells. For Panc-1 and MiaPaCa cells, MMP-9 activities were decreased under both low and high glucose conditions (Figure $4 \mathrm{a}, P<0.05$; Figure $4 \mathrm{~b}, P<0.01$ ). V-ATPase inhibition had modest effects on BXPC3 cells (Figure 4c) under low glucose conditions only. These findings were corroborated in Panc-1 cells with shRNA knockdown of the $\mathrm{V}_{1} \mathrm{E}$ subunit (Figure $4 \mathrm{~d}$ ). These cells showed significantly decreased MMP-9 activity that corresponded to the extent of $V_{1} E$ subunit knockdown. These results indicate pancreatic cancer cells with PM localization of the v-ATPase are susceptible to MMP-9 activities that depend on $\mathrm{v}$-ATPase function.
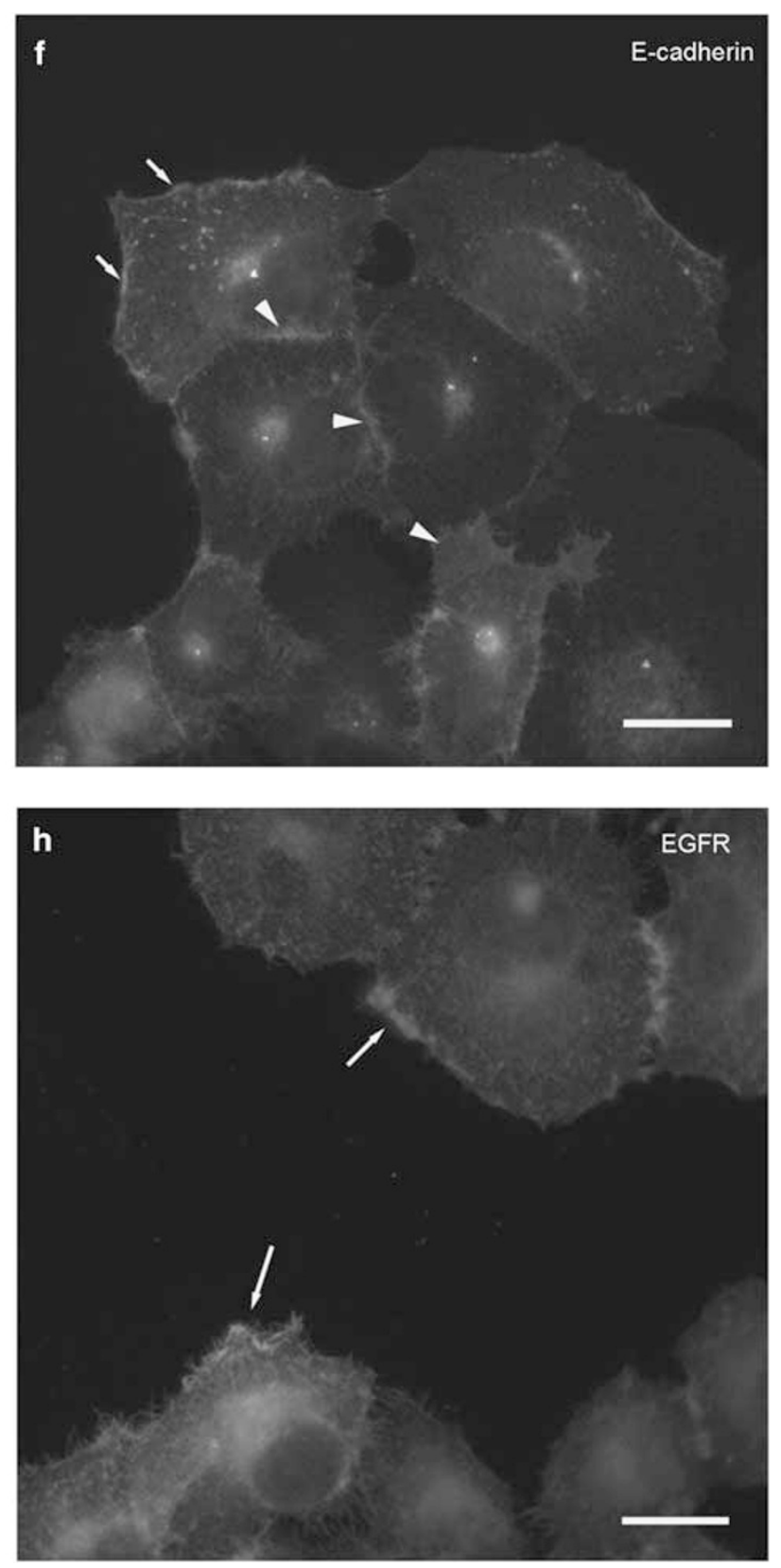

V-ATPase inhibition yielded different effects on MMP-2 activity. MMP-2 activity was evident in the CM of Panc-1 cells, but barely detectable in MiaPaCa and BXPC3 lines (data not shown). In Panc-1 cells, the intermediately active $(66 \mathrm{kDa})$ zymogen and the fully active $(62 \mathrm{kDa})$ protease forms (Figure 4e) could be differentiated. V-ATPase inhibition increased the most active forms of MMP-2 significantly more than under control conditions $(P<0.001)$. Similar findings on MMP-2 activation were obtained using the related v-ATPase inhibitor, bafilomycin $(50 \mathrm{nM}$ ), (Supplementary Figure 4). These results point to the different regulation and activation of MMP-2 versus MMP-9 precursor 

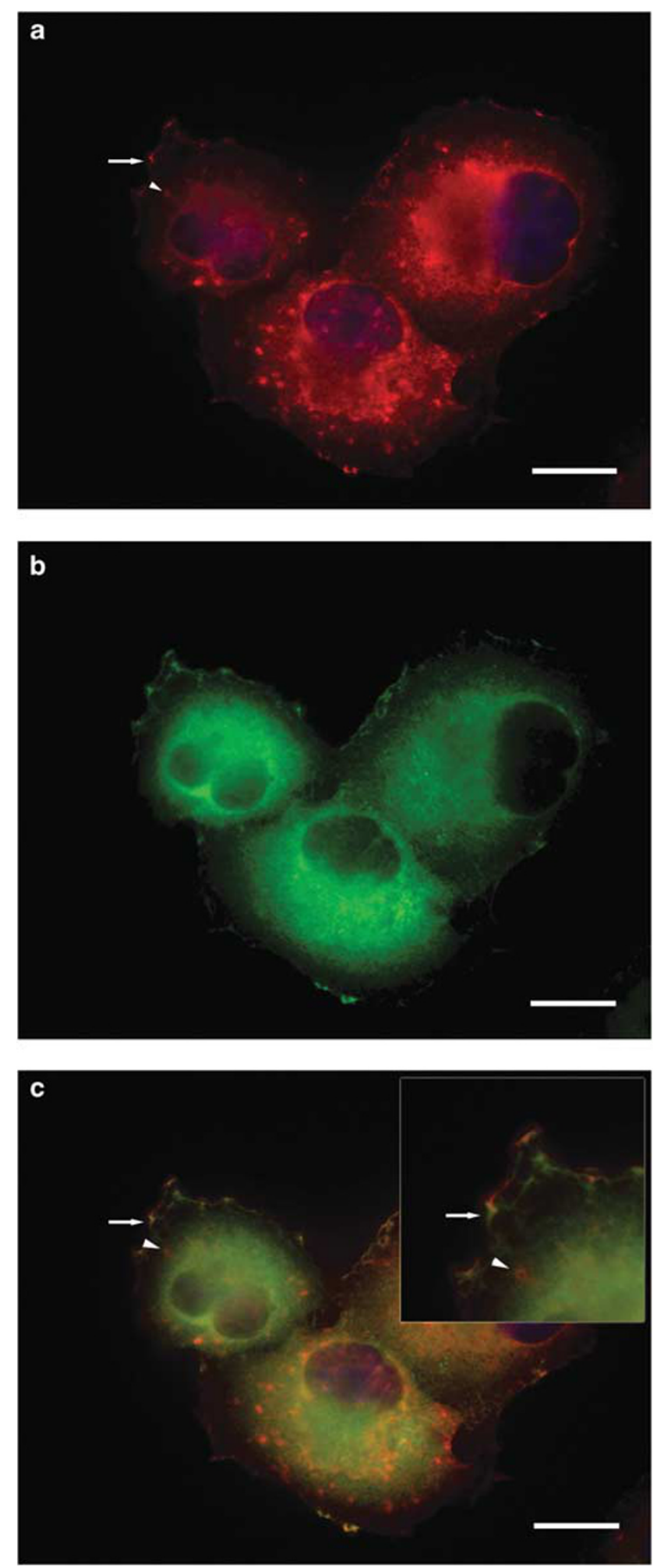

Figure 3 Panc-1 cells show colocalization of vacuolar-ATPase (v-ATPase) with cortactin, a component of the cellular invasion apparatus. Immunofluorescent (IF) images of the $\mathrm{V}_{1} \mathrm{E}$ isoform ( $\mathbf{a}$, red) and cortactin (b, green) in Panc-1 cells reveal that in those cells with plasma membrane, v-ATPase localization proximity to known components of the cellular invasion apparatus such as cortactin was observed. Non-overlapping intracellular areas of $\mathrm{V}_{1} \mathrm{E}$ localization are shown (inset, red circular structures, arrowhead) adjacent to merged areas (c, inset, yellow). forms, and are consistent with a previous report on enhanced MMP-2 activities in melanoma cells treated with bafilomycin. $^{31}$

\section{Invasion and Cellular Degradation are V-ATPase Dependent}

The functional effects of v-ATPase inhibition on cell invasion and migration were assessed. An agar-based assay showed that MiaPaCa cells were able to invade and this was inhibited in the presence of concanamycin (Figure 5, top). Furthermore, concanamycin treatments also inhibited migration across a wound (Figure 5, bottom; $P<0.05$ ), with 21.9 and $35.1 \%$ greater diameter compared with controls under low and high glucose conditions, respectively. Panc-1 cells showed no differences in invasion in the presence of concanamycin (data not shown), a finding that may reflect the complex effects of concanamycin treatment on MMP activities: decreased MMP-9, but increased MMP-2 active isoforms.

\section{DISCUSSION}

This study illustrated that the cellular distribution of v-ATPase in human pancreatic cancer tissues may influence cancer cell activity as polarity is lost and expression was increased with advancing malignant features. In fact, striking differences in v-ATPase polarity and staining intensity distinguished early from advanced PanIN lesions. Invasive pancreatic cancers and metastatic lesions meanwhile showed uniformly diffuse and intense v-ATPase staining. These findings indicate that elevated expression and loss of $\mathrm{v}$-ATPase polarity may be key steps in modulating the tumor microenvironment, thereby providing a clinical correlate to previous in vitro work in breast cancer cells that identified v-ATPase expression as a marker of cancer cell aggressiveness. ${ }^{11}$

A previous study in human pancreatic cancer specimens compared mRNA levels and immunolabeling of the v-ATPase $\mathrm{V}_{0} \mathrm{C}$ subunit in PDAC in relation to precursor lesions and benign cystic tumors. ${ }^{32}$ This study indicated that the mRNA levels of this subunit were increased in PDAC eightfold over normal pancreas. Similar to the results reported here, the intensity of v-ATPase expression was highest in PDAC. The absence of positive staining in non-invasive cancers or in benign cystic neoplasms in this previous study is a notable difference from our results. This study found that PanIN lesions showed prominent v-ATPase labeling with a clear loss of polarity that coincided with increasing malignant features. Our current results show a unique pattern to $\mathrm{v}$-ATPase $\left(\mathrm{V}_{1} \mathrm{E}\right.$ subunit) labeling within PDAC precursors, which suggests an early role for v-ATPase function in cancer cell homeostasis and invasive capacity as corroborated by previous literature. ${ }^{11,19,33}$ Future evaluation of v-ATPase expression in human cancer sections using standardized antibodies and techniques may be necessary to resolve these differences.

Other studies have shown that the v-ATPase on PMs contributes to acidification of the extracellular space, which promotes invasive properties. ${ }^{11,34}$ Targeted inhibition of the 
$\mathrm{V}_{0} \mathrm{c}$ subunit led to diminished MMP-2 expression and reduced hepatocellular carcinoma growth in an animal model, indicating that the therapeutic potential of inhibiting the $\mathrm{v}$-ATPase may be due in part to reducing MMP-2 activity. ${ }^{34,35}$ However, whether these findings are relevant to other types of cancers and other MMPs is unclear. We showed that the v-ATPase is present on PMs of Panc-1 cells; these cell lines have been described to have invasive potential in vivo. ${ }^{28}$ In Panc-1 cells, the v-ATPase colocalizes with cortactin, a component of the cellular invasion apparatus implicated in focal MMP-9 release. ${ }^{20,21}$ Significantly, MMP-9 activity was reduced with v-ATPase blockade in three pancreatic cancer cell lines, but was least affected in BXPC3 cells, which showed little v-ATPase localization. Targeted inhibition of the $\mathrm{V}_{1} \mathrm{E}$ subunit using shRNA constructs confirmed these findings in Panc-1 cells. Thus, specific pancreatic cancer cells display v-ATPase PM localization and MMP-9 activities that are v-ATPase dependent.

In contrast to MMP-9, concanamycin and bafilomycin inhibition of the v-ATPase increased the fully active MMP-2 isoform. A potential explanation for this difference may be the differential regulation and activation of MMP-2 and -9 precursors. MT1-MMP, a cell surface activator of MMP-2, is rapidly and constitutively downregulated through a v-ATPase-dependent degradation process. ${ }^{31,36}$ V-ATPase blockade results in increased levels of active MT1-MMP on the cell surface, thereby amplifying MMP-2 activities, which is consistent with our findings. ${ }^{31,37}$ These findings indicate that
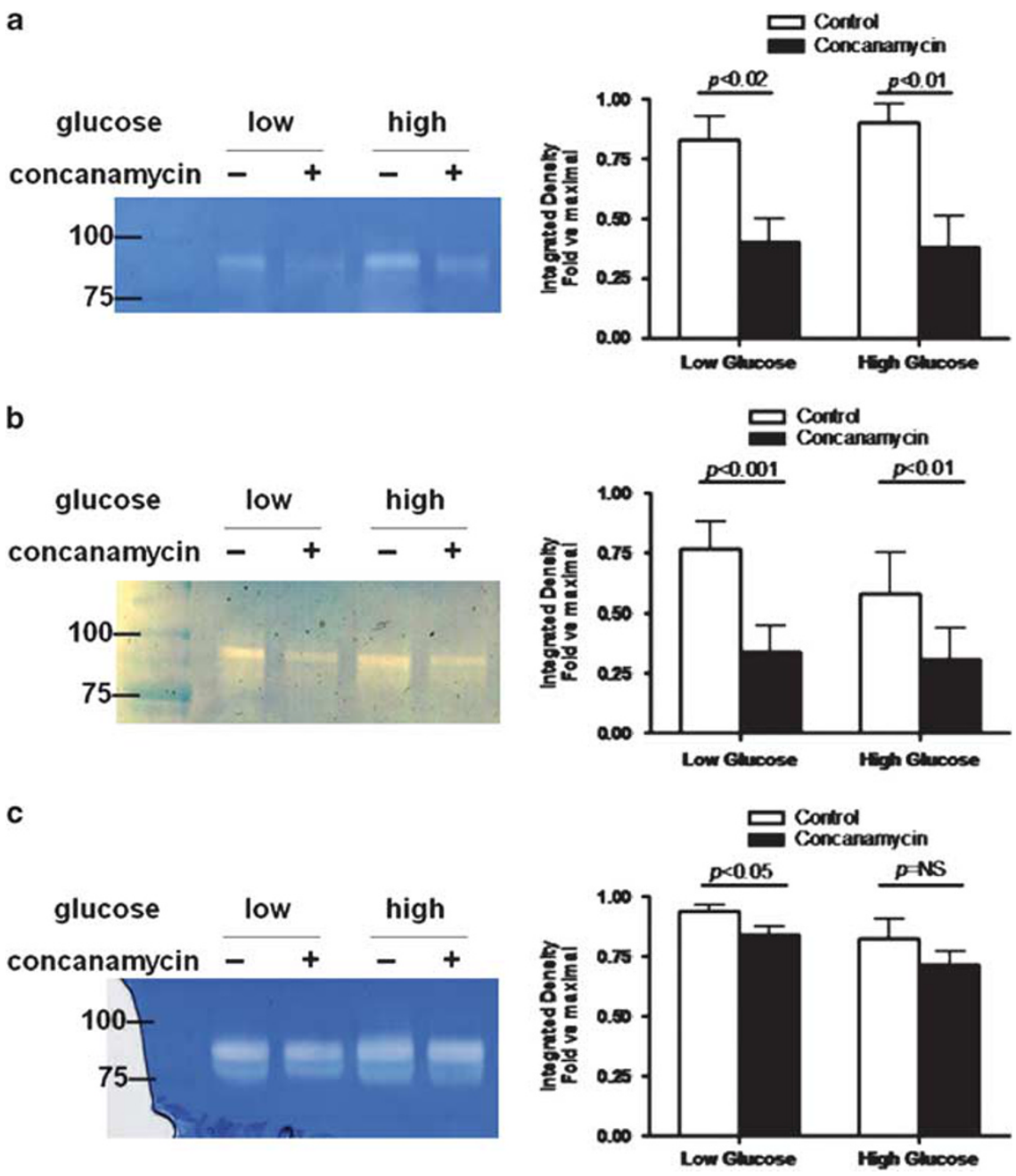

Figure 4 Pancreatic cancer cell-derived matrix metalloproteinase (MMP)-9, but not MMP-2, displays reduced activities after vacuolar-ATPase (v-ATPase) blockade. Effects of v-ATPase inhibition on MMP-9 activity using concanamycin in (a) Panc-1, (b) MiaPaCa, and (c) BXPC3 cells are shown. In low glucose conditions, concanamycin reduced MMP-9 activity more than twofold in Panc-1 and MiaPaCa cells, and to a lesser extent in BXPC3 cells. In high glucose conditions, concanamycin also reduced MMP-9 activity in Panc-1 and MiaPaCa cells, but did not have a significant effect on BXPC3 cells. (d) Targeted knockdown of $\mathrm{V}_{1} \mathrm{E}$ by short-hairpin RNA (shRNA) constructs E1 and E2 results in decreased MMP-9 activities. (e) V-ATPase inhibition with concanamycin results in increased activity of the fully activated ( $62 \mathrm{kDa}$ ) MMP-2 isoform. Representative zymogram of Panc-1 CM was shown (top panel). Higher MMP-2 activity of the intermediately active $(66 \mathrm{kDa})$ form under high glucose conditions was seen (middle panel), whereas the $62 \mathrm{kDa}$ form was significantly higher in the presence of concanamycin under both low and high glucose conditions (upper panel). 
d

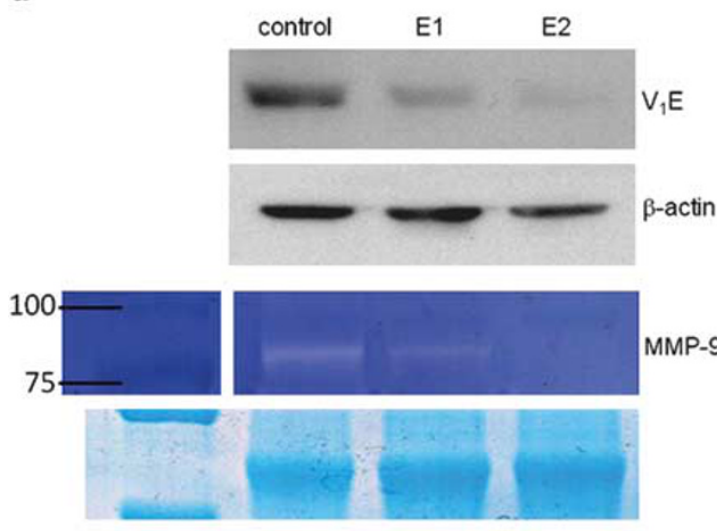

e

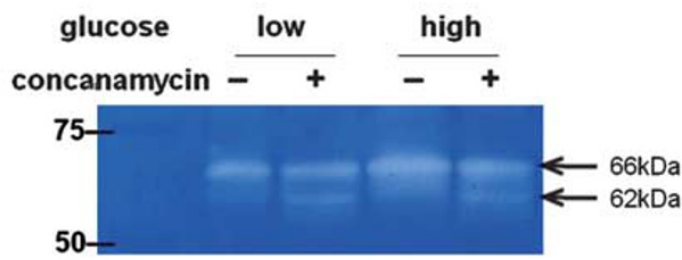

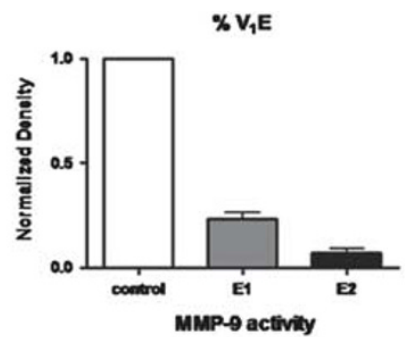
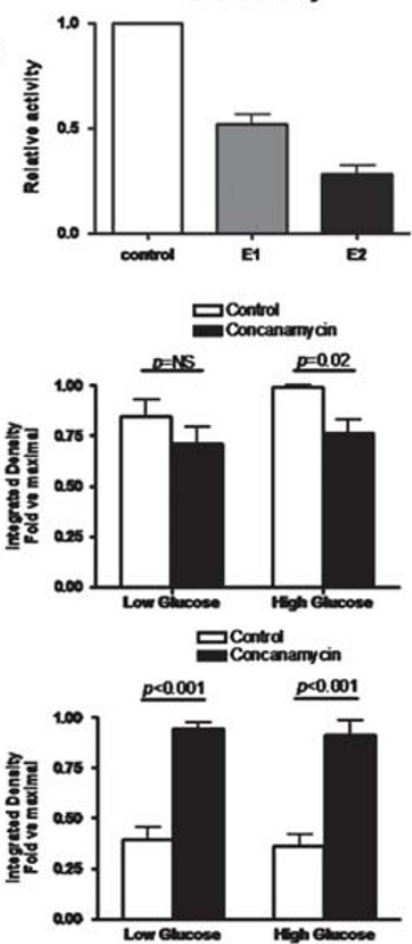

Figure 4 Continued.

potential targeting of the v-ATPase in cancer studies may have indirect (and unintended) effects on regulators of MMP activation that could enhance, rather than block, specific protease activities.

As MMP-2 activation with concanamycin treatments reflects the ability of intact intracellular degradation pathways that are v-ATPase dependent, these studies using chemical $\mathrm{v}$-ATPase inhibitors do not allow us to discern the relative contributions of extracellular versus intracellular proton flux. Future experiments targeting the specific subunits associated with PM localization may help to provide additional evidence supporting a role for v-ATPase-mediated extracellular acidification.

Although we have shown that the v-ATPase can modulate MMP activities in cancer cells, this transporter can affect other cellular responses that may be relevant to cancer biology. For instance, v-ATPases may mediate resistance to chemotherapeutic agents. Tumor cells, when exposed to chemotherapeutic drugs, show transcriptional promoter activity that leads to the induction of specific $\mathrm{v}$-ATPase levels. ${ }^{38,39}$ This induction precludes cancer cell apoptosis in response to chemotherapy, showing that v-ATPase expression may be a protective mechanism against chemically induced apoptosis. Combined application of chemotherapy and a v-ATPase inhibitor restored the ability of these agents to cause cancer cell apoptosis. ${ }^{39}$ Thus, in addition to effects on MMP activities, targeting v-ATPases could also enhance the sensitivity of cancer cells to chemotherapeutics.

Accumulating evidence also points to the importance of cell surface v-ATPase function in non-malignant processes. In renal tubular epithelium, the v-ATPase is vital for urine acidification. Osteoclasts are enriched with specific v-ATPase isoforms at the ruffled border, which enables an optimal $\mathrm{pH}$ environment for proteases to degrade matrix. Endothelial cells adjacent to an induced wound display inducible and prominent PM v-ATPase localization and activity. ${ }^{10}$ Endothelial cells obtained from diabetic rat models, however, display significantly reduced cell surface v-ATPase activity and show a functional impairment in migratory behavior. ${ }^{40}$ These findings indicate that physiological and wound-repair processes likely require PM v-ATPase activity, possibly due to the effects on MMPs. 
control

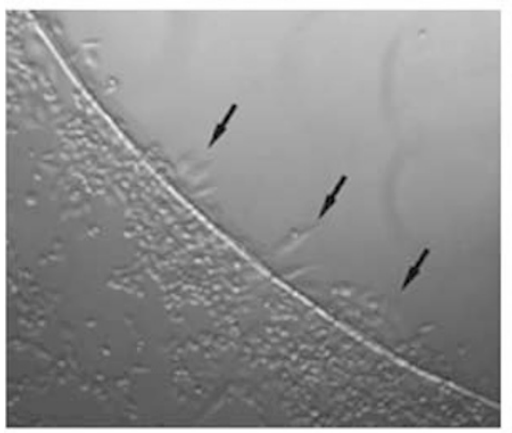

concanamycin

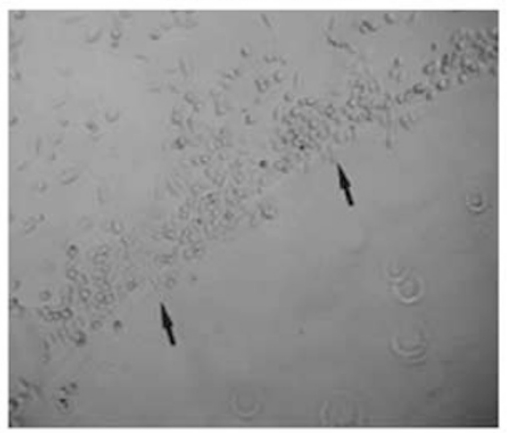

$p<0.01$

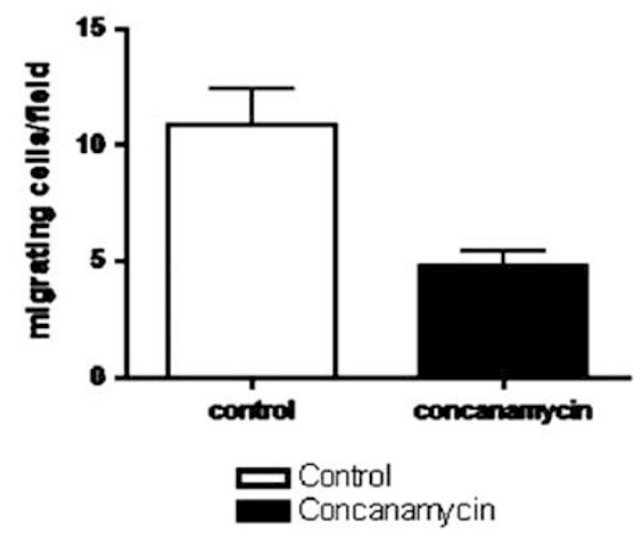

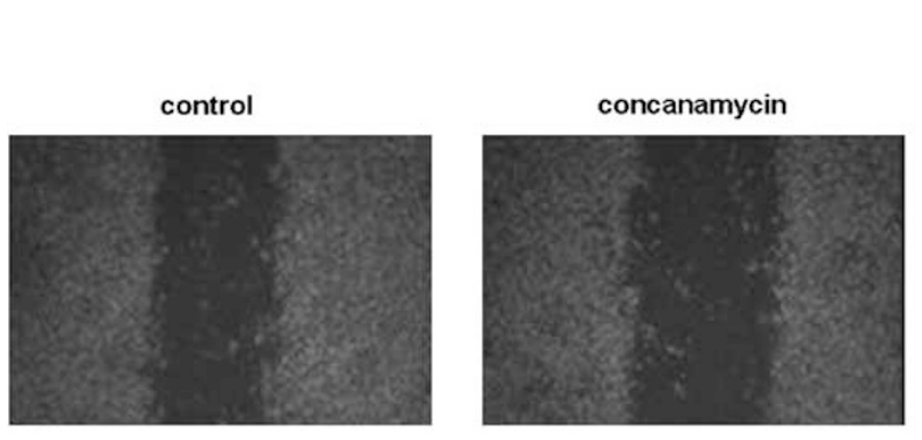

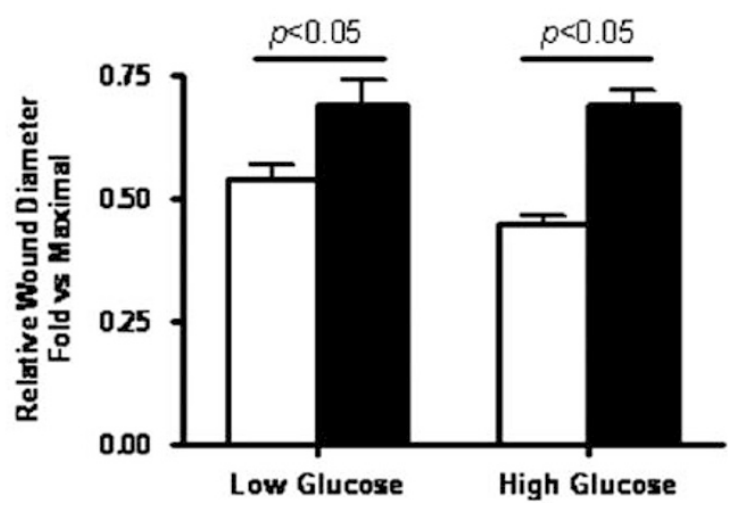

Figure 5 Vacuolar-ATPase (v-ATPase) function is necessary for pancreatic cancer cell migration and invasion. (Top) Control MiaPaCa cells (right) migrate into an agar disk, whereas adding the v-ATPase inhibitor concanamycin to the medium inhibits their migration. (Bottom) Scratch wound migration assay shows larger relative wound diameter after concanamycin compared with control MiaPaCa cells, showing that v-ATPase blockade inhibits migration.

In summary, v-ATPase staining of human pancreatic malignancy, ranging from PanIN lesions to PDAC, showed a marked loss of polarity and increased intensity with increasing tumor invasiveness. These changes were seen in PanIN lesions, suggesting a role for the v-ATPase in early stages of malignant transformation. Inhibiting v-ATPase function decreased MMP-9 activities, but increased MMP-2 activation in vitro. These results indicate that the v-ATPase plays a complex role in regulating MMPs and the pancreatic cancer phenotype.

Supplementary Information accompanies the paper on the Laboratory Investigation website (http://www.laboratoryinvestigation.org)

\section{ACKNOWLEDGEMENT}

This work was supported by a VA CDA Award, National Pancreas Foundation (CC); NIH CA133346 (AJK); NSF Graduate Research Fellowship (CCM); R01 CA064238 (SEC); and RO1 DK54021 (FSG).

\section{DISCLOSURE/CONFLICT OF INTEREST}

The authors declare no conflict of interest.

1. Bertout JA, Patel SA, Simon MC. The impact of $\mathrm{O}_{2}$ availability on human cancer. Nat Rev Cancer 2008;8:967-975.
2. Hinton $A$, Bond S, Forgac M. V-ATPase functions in normal and disease processes. Pflugers Arch 2009:457:589-598.

3. Sennoune SR, Martinez-Zaguilan R. Plasmalemmal vacuolar $\mathrm{H}^{+}$ATPases in angiogenesis, diabetes and cancer. J Bioenerg Biomembr 2007;39:427-433.

4. Nelson N. Structure, molecular genetics, and evolution of vacuolar $\mathrm{H}+-$ ATPases. J Bioenerg Biomembr 1989;21:553-571.

5. Arvan P, Castle JD. Isolated secretion granules from parotid glands of chronically stimulated rats possess an alkaline internal $\mathrm{pH}$ and inwarddirected $\mathrm{H}^{+}$pump activity. J Cell Biol 1986;103:1257-1267.

6. Orci L, Ravazzola M, Amherdt M, et al. Conversion of proinsulin to insulin occurs coordinately with acidification of maturing secretory vesicles. J Cell Biol 1986;103:2273-2281.

7. Beyenbach KW, Wieczorek $\mathrm{H}$. The V-type $\mathrm{H}^{+}$ATPase: molecular structure and function, physiological roles and regulation. J Exp Biol 2006;209:577-589.

8. Wagner $\mathrm{CA}$, Finberg $\mathrm{KE}$, Breton $\mathrm{S}$, et al. Renal vacuolar H+-ATPase. Physiol Rev 2004;84:1263-1314.

9. Nanda A, Brumell JH, Nordstrom $\mathrm{T}$, et al. Activation of proton pumping in human neutrophils occurs by exocytosis of vesicles bearing vacuolar-type $\mathrm{H}^{+}$-ATPases. J Biol Chem 1996; 271:15963-15970.

10. Rojas JD, Sennoune SR, Maiti $D$, et al. Vacuolar-type $H^{+}$-ATPases at the plasma membrane regulate $\mathrm{pH}$ and cell migration in microvascular endothelial cells. Am J Physiol Heart Circ Physiol 2006;291: H1147-H1157.

11. Sennoune SR, Bakunts $\mathrm{K}$, Martinez GM, et al. Vacuolar $\mathrm{H}^{+}$-ATPase in human breast cancer cells with distinct metastatic potential: distribution and functional activity. Am J Physiol Cell Physiol 2004;286:C1443-C1452. 
12. Nishi T, Forgac $M$. The vacuolar $(\mathrm{H}+)$-ATPases-nature's most versatile proton pumps. Nat Rev Mol Cell Biol 2002;3:94-103.

13. Toyomura T, Oka T, Yamaguchi $C$, et al. Three subunit a isoforms of mouse vacuolar $\mathrm{H}(+)$-ATPase. Preferential expression of the a3 isoform during osteoclast differentiation. J Biol Chem 2000;275:8760-8765.

14. Toyomura T, Murata $\mathrm{Y}$, Yamamoto $\mathrm{A}$, et al. From lysosomes to the plasma membrane: localization of vacuolar-type $\mathrm{H}^{+}$-ATPase with the a3 isoform during osteoclast differentiation. J Biol Chem 2003;278: 22023-22030.

15. Frattini A, Orchard PJ, Sobacchi C, et al. Defects in TCIRG1 subunit of the vacuolar proton pump are responsible for a subset of human autosomal recessive osteopetrosis. Nat Genet 2000;25:343-346.

16. Kornak U, Schulz A, Friedrich W, et al. Mutations in the a3 subunit of the vacuolar $\mathrm{H}(+)$-ATPase cause infantile malignant osteopetrosis. Hum Mol Genet 2000;9:2059-2063.

17. Li YP, Chen W, Liang Y, et al. Atp6i-deficient mice exhibit severe osteopetrosis due to loss of osteoclast-mediated extracellular acidification. Nat Genet 1999;23:447-451.

18. Frattini A, Blair HC, Sacco MG, et al. Rescue of ATPa3-deficient murine malignant osteopetrosis by hematopoietic stem cell transplantation in utero. Proc Natl Acad Sci USA 2005;102:14629-14634.

19. Hinton A, Sennoune SR, Bond $S$, et al. Function of a subunit isoforms of the V-ATPase in $\mathrm{pH}$ homeostasis and in vitro invasion of MDA-MB231 human breast cancer cells. J Biol Chem 2009;284: 16400-16408.

20. Clark ES, Weaver AM. A new role for cortactin in invadopodia: regulation of protease secretion. Eur J Cell Biol 2008;87:581-590.

21. Clark ES, Whigham AS, Yarbrough WG, et al. Cortactin is an essential regulator of matrix metalloproteinase secretion and extracellular matrix degradation in invadopodia. Cancer Res 2007;67: 4227-4235.

22. Sautin YY, Lu M, Gaugler A, et al. Phosphatidylinositol 3-kinasemediated effects of glucose on vacuolar $\mathrm{H}^{+}$-ATPase assembly translocation, and acidification of intracellular compartments in renal epithelial cells. Mol Cell Biol 2005;25:575-589.

23. Sumner JP, Dow JA, Earley FG, et al. Regulation of plasma membrane V-ATPase activity by dissociation of peripheral subunits. J Biol Chem 1995;270:5649-5653.

24. Chung C, Shugrue C, Nagar A, et al. Ethanol exposure depletes hepatic pigment epithelium-derived factor, a novel lipid regulator. Gastroenterology 2009;136:331-340 e2.

25. Visse $R$, Nagase $H$. Matrix metalloproteinases and tissue inhibitors of metalloproteinases: structure, function, and biochemistry. Circ Res 2003;92:827-839.
26. Theret N, Musso $\mathrm{O}$, L'Helgoualc' $\mathrm{h} A$, et al. Activation of matrix metalloproteinase- 2 from hepatic stellate cells requires interactions with hepatocytes. Am J Pathol 1997;150:51-58.

27. Wiggins $H$, Rappoport J. An agarose spot assay for chemotactic invasion. Biotechniques 2010:48:121-124.

28. Suemizu H, Monnai M, Ohnishi $Y$, et al. Identification of a key molecular regulator of liver metastasis in human pancreatic carcinoma using a novel quantitative model of metastasis in NOD/SCID/gammacnull (NOG) mice. Int J Oncol 2007;31:741-751.

29. Weaver AM. Invadopodia: specialized cell structures for cancer invasion. Clin Exp Metast 2006;23:97-105.

30. Clark ES, Brown B, Whigham AS, et al. Aggressiveness of HNSCC tumors depends on expression levels of cortactin, a gene in the 11 q13 amplicon. Oncogene 2009;28:431-444.

31. Maquoi E, Peyrollier K, Noel A, et al. Regulation of membrane-type 1 matrix metalloproteinase activity by vacuolar $\mathrm{H}^{+}$-ATPases. Biochem J 2003;373:19-24.

32. Ohta $T$, Numata $M$, Yagishita $H$, et al. Expression of $16 \mathrm{kDa}$ proteolipid of vacuolar-type $\mathrm{H}(+)$-ATPase in human pancreatic cancer. $\mathrm{Br} \mathrm{J}$ Cancer 1996;73:1511-1517.

33. Fais $\mathrm{S}$, De Milito $\mathrm{A}$, You $\mathrm{H}$, et al. Targeting vacuolar $\mathrm{H}^{+}$-ATPases as a new strategy against cancer. Cancer Res 2007;67:10627-10630.

34. Lu X, Qin W, Li J, et al. The growth and metastasis of human hepatocellular carcinoma xenografts are inhibited by small interfering RNA targeting to the subunit ATP6L of proton pump. Cancer Res 2005;65:6843-6849.

35. Kato $\mathrm{Y}$, Lambert $\mathrm{CA}$, Colige $\mathrm{AC}$, et al. Acidic extracellular $\mathrm{pH}$ induces matrix metalloproteinase- 9 expression in mouse metastatic melanoma cells through the phospholipase D-mitogen-activated protein kinase signaling. J Biol Chem 2005;280:10938-10944.

36. Poincloux R, Lizarraga F, Chavrier P. Matrix invasion by tumour cells: a focus on MT1-MMP trafficking to invadopodia. J Cell Sci 2009; 122:3015-3024.

37. Zucker S, Hymowitz M, Conner CE, et al. Rapid trafficking of membrane type 1-matrix metalloproteinase to the cell surface regulates progelatinase a activation. Lab Invest 2002;82:1673-1684.

38. Murakami T, Shibuya I, Ise T, et al. Elevated expression of vacuolar proton pump genes and cellular $\mathrm{PH}$ in cisplatin resistance. Int J Cancer 2001;93:869-874.

39. Torigoe $\mathrm{T}$, Izumi $\mathrm{H}$, Ishiguchi $\mathrm{H}$, et al. Enhanced expression of the human vacuolar $\mathrm{H}^{+}$-ATPase $\mathrm{c}$ subunit gene (ATP6L) in response to anticancer agents. J Biol Chem 2002;277:36534-36543.

40. Rojas JD, Sennoune SR, Martinez GM, et al. Plasmalemmal vacuolar $\mathrm{H}^{+}$ATPase is decreased in microvascular endothelial cells from a diabetic model. J Cell Physiol 2004;201:190-200. 